\title{
The Morphology of Barred Galaxies
}

\author{
Ronald Buta \\ Department of Physics and Astronomy, University of Alabama, Box \\ 870324, Tuscaloosa, AL 35487
}

\begin{abstract}
.
In this review, the morphology of barred galaxies at optical wavelengths is discussed, focussing mainly on early to intermediate revised Hubble types in the range $\mathrm{SO}^{+}$to Sbc. Previous work by Hubble, Sandage, de Vaucouleurs, Vorontsov-Velyaminov, and Kormendy is described. The primary emphasis is on ring phenomena, and morphological results from the Catalog of Southern Ringed Galaxies are summarized.
\end{abstract}

\section{Introduction}

Barred galaxies easily present some of the most complex morphologies among the population of normal disk galaxies. Bars play an important role in shaping the gravitational potential in galaxies, and as a consequence barred galaxies are rich in morphological features that are thought to be directly caused by the bar. More than $50 \%$ of all disk galaxies appear to have a bar in some form, either as a standard SB-type bar, an oval distortion, or a secondary bar. Understanding the connection between these perturbations and other aspects of galaxy morphology is one of the current challenges of modern astrophysics.

In this review, I would like to describe barred galaxy morphology to help set some of the groundwork for the rest of this conference, because morphology can provide some important clues to the dynamics and evolution of these objects. The review is intended to complement the previous excellent articles covering the same topic by Kormendy $(1979,1981,1982 \mathrm{a})$, with an emphasis on inner, outer, and nuclear ring and pseudoring phenomena. Let me first discuss some of the historical aspects of the subject, and then I will cover the more recent results.

\section{Early Observations}

One of the earliest recognitions of the bar form was made visually by colleagues of W. Parsons (Third Earl of Rosse) with the 1.8-m speculum reflector at Birr Castle in Ireland. Observations in 1858 and 1867 revealed the bar and part of the inner ring of $\mathrm{NGC} 4725$; it was, in fact, noted that this object presented the general appearance of a ship's anchor. The first photographic recognition of barred spirals as a class is credited to Curtis (1918), who called them $\phi$-type spirals because of a general appearance to the Greek letter $\phi$. Hubble (1926) later suggested that the term $\theta$-type spiral was more appropriate for these objects 
because the bar never extended beyond the inner spiral arms. Since Greek letters were inconvenient for catalogs, he coined the term "barred spiral" and suggested the contracted symbol "SB". He also recognized that apart from the bar, these spirals could be placed on a sequence similar to the "normal" (or nonbarred) spirals. Most of the original SBa galaxies did not have spiral structure, and the barred spiral sequence was revised by Hubble (1936) after the discovery of $\mathrm{S} 0$ galaxies.

Though Hubble had later appreciated that some galaxies might be intermediate between a true barred and a true nonbarred spiral (see Sandage 1961), it was de Vaucouleurs (1959) who truly recognized the continuity of the bar characteristic. He recommended dropping the term "normal" spiral and replacing it with "ordinary" spiral. The ordinary spirals would not simply be "S" but "SA", such that those galaxies judged to be of intermediate bar characteristic could be given the combined symbol "SAB". The sequence SA-SAB-SB is not necessarily directly connected to a continuously varying true "bar strength". On average there may be some correlation, but the scatter would be large.

The detailed nature of the de Vaucouleurs revised Hubble classification system allows it to give the best indication of the frequency of bars that can be obtained from blue-light images. De Vaucouleurs (1963) classified 1500 bright galaxies from large-scale plates. From 994 spirals, he found that $31 \%$ are SA, $28 \%$ are $\mathrm{SAB}$, and $37 \%$ are $\mathrm{SB}$. Thus, $65 \%$ of bright galaxies have a recognizable bar or bar-like feature in blue light. In the near-infrared, the figure is likely to be considerably higher.

The classification of barred S0 (or SB0) galaxies provides a good contrast between Hubble's revised Hubble system and de Vaucouleurs' revised Hubble system. The Hubble revision is beautifully illustrated by Sandage \& Bedke (1994), where the classification of barred S0's depends entirely on the character of the bar. $\mathrm{SB}_{1}$ galaxies have a barely differentiated oval in the bulge-dominated region; $\mathrm{SBO}_{2}$ galaxies have a bar of the "ansae"-type and often are associated with outer rings; and $\mathrm{SB}_{3}$ galaxies have a complete bar crossing the inner disk region. The de Vaucouleurs classification of early and late SB0's, through the notations $\mathrm{SB}^{-}, \mathrm{SB0}^{\circ}$, and $\mathrm{SB}^{+}$, depends mainly on the development of disk structure, such as weak spiral patterns or rings. Barred S0's with strong rings are usually recognized as of type $\mathrm{SB} 0^{+}$.

Barred galaxies are also recognized in the descriptive classification system of the Morphological Catalog of Galaxies (Vorontsov-Velyaminov et al. 1962$1968=\mathrm{MCG}$ ). Bars are denoted $\mathrm{B}^{-}, \mathrm{B}, \mathrm{BB}$, or $\mathrm{BBB}$ in order of increasing relative length. Objects with a strong central region are denoted as $\mathrm{N} ; 2 \mathrm{~B}$, meaning the bar is two extensions out from the nuclear region.

\section{Components of Barred Galaxies}

The number of "cells" in the de Vaucouleurs revised Hubble system is about 120. In part because of this large number, Kormendy (1979) proposed another approach to morphology. Kormendy suggested that galaxies can be broken into a small number of "building blocks" or "distinct components." Some of these components may form separately, independent of the rest of a galaxy, while others may form from primary components via internal interactions. The primary 


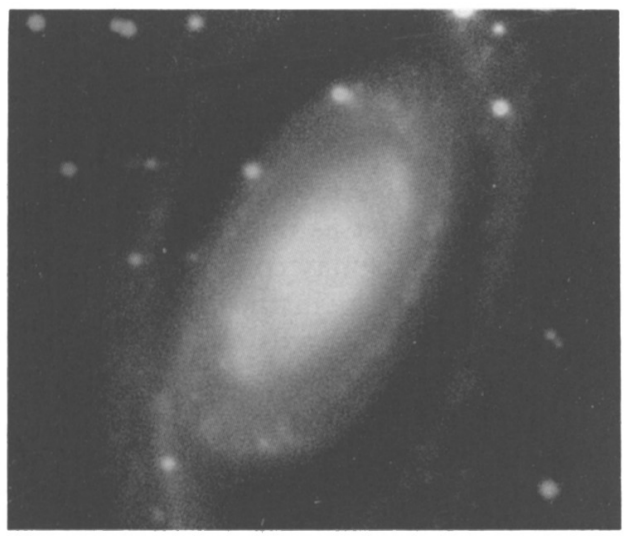

Figure 1. The unusual "ansae-type" bar in NGC 7098 ( $B$-band).

components are bars, spheroids, and disks. Other components include lenses, defined to be elliptical-shaped features between the spheroid and disk which have a shallow brightness gradient interior to a sharp edge; and inner and outer rings. Among SB0-SBa galaxies, 54\% were found to have lenses, while among SBab-SBc galaxies, $76 \%$ were found to have inner rings. Thus, lenses and inner rings are important features of barred galaxies.

The value of Kormendy's study is that it provided some physical insight into barred galaxies that the basic classification systems could not provide. The main insight was into the nature of lenses. Of 20 galaxies in his sample with a strong bar and lens, the bar fills the lens in one dimension in 17 cases. This suggested to Kormendy that lenses result from slow secular dissolution of bars. Since lenses are most prominent in early-type barred galaxies, an interaction with the bulge component was thought likely. Theoretical aspects of rings and lenses are discussed further by Combes, these proceedings.

\section{The Morphology of Bars and Barred Galaxy Bulges}

Bars show a wide range of morphologies. Regular bars, such as those in NGC 1300 and 1433, are the conventional types. Near stage Sb, regular bars often have prominent linear dust lanes (Sandage 1961) that are clearly revealed on color index maps even when weak in blue light. In contrast, many bars in early-type galaxies have bright enhancements ("ansae") near the ends that give them the appearance of a dumbbell. Ansae bars are also found in early-to-intermediate Hubble type spirals such as NGC 7098 and ESO 509-98. In NGC 7098, the ansae are nearly straight bright arcs, so that the bar actually appears shaped like a parallelogram, not a dumbbell (see Figure 1). A color index map shown by Buta (1995) reveals no color contrast associated with these ansae compared to the rest of the bar or the surrounding region. Ansae therefore appear to be largely stellar-dynamical in nature.

That bars possess a general boxy character was shown by Athanassoula et al. (1990). This is especially true in early-type barred galaxies. The boxiness 
can be quantified and connected to theoretical models. NGC 7098 is an extreme example of a boxy, somewhat fat bar.

Oval bar-like distortions are evident in many galaxies. A prime example is NGC 210, shown in Sandage (1961) and Sandage \& Bedke (1994). Regular bars and ovals co-exist in some galaxies. An oval may be classified as a lens or even a ring around a bar. Ovals can be just as important to the dynamics of a galaxy as a conventional bar, because ovals may contain a great deal more mass than a conventional bar. It is possible for the intrinsic major axis of an oval lens to be significantly misaligned with the major axis of a bar (see section 6.2). This kind of configuration suggests that some lenses are not a result of the slow dissolution of a bar.

The bulges of barred S0 galaxies can be very different from what is seen in $\mathrm{SA}$ galaxies. Kormendy (1982b) brought attention to the frequent misalignment between bulge isophotes and disk isophotes in SB0 galaxies, and noticed that several SB bulges were oriented perpendicular to the bar. Even when an SB bulge has isophotes that are aligned with the disk, the isophote shapes compared to the disk rule out an oblate intrinsic shape. Kormendy suggested that these characteristics implied that some SB bulges are triaxial. The idea has been supported by kinematic studies, which have indicated that the bulges of SB galaxies rotate more rapidly and are more flattened than those in SA galaxies. Triaxial bulges are discussed further by Friedli, these proceedings.

\section{Bar Dust Lanes}

Color index maps reveal interesting dust lane patterns in barred and weaklybarred galaxies. These lanes are often connected with nuclear star formation in the form of nuclear rings or blue nuclei. The morphology of bar dust lanes has been considered by Athanassoula (1992), who demonstrated how bar strength and the existence of an ILR help to shape these features. She identified three major dust lane types among the regular bars: type $a$ lanes are most linear while type $c$ lanes are most curved. Type $b$ lanes are straight along the bar but curve in the vicinity of the core, hence are intermediate between type $a$ and $c$ lanes. Weaker bars appear to have the most curved or complex dust lanes. There are also some regular but unusual patterns that merit further study. For example, Benedict et al. (1992) have found that the dust lanes in the bar of NGC 4314 curve around only half-way out along the bar length, causing them to speculate that the bar of this galaxy extends well beyond corotation, an unexpected result. Other cases are described by Buta \& Crocker (1991).

\section{Rings and Pseudorings as Probes of Barred Galaxy Dynamics}

The association between bars and the components known as rings, pseudorings, and lenses provides an important link between morphology and processes of secular evolution and internal dynamics. In 1979 I began a systematic study of "ringed galaxies" to learn more about the photometric and kinematic properties of such systems (1984). This work and the works of Schwarz $(1979,1981)$, Kormendy (1979), and de Vaucouleurs (1956) stimulated a survey called the Catalog of Southern Ringed Galaxies (CSRG, Buta 1995). Begun in 1984, the CSRG 


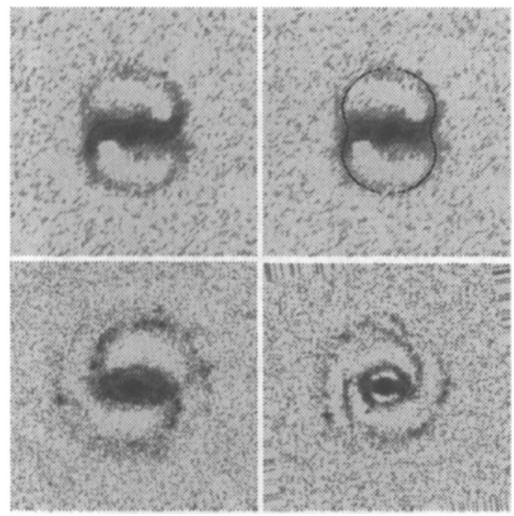

Figure 2. Illustration of three galaxies with $\mathrm{R}_{1}^{\prime}$ or $\mathrm{R}_{2}^{\prime}$ outer pseudorings. Upper panels: ESO 287-56 with oval of Cassini superposed at right; Lower panels: ESO 325-28 (left); ESO 577-3 (right). These images have had a median-smoothed background subtracted. ESO 287-56 and ESO 577-3 have been deprojected according to the shape of an outer isophote. Field stars have been removed.

is a comprehensive compilation of angular diameters, axis ratios, and relative major axis position angles of inner and outer rings and pseudorings in 3,692 galaxies south of declination $-17^{\circ}$. The principal objective of the survey was to use statistics of apparent axis ratios and bar/ring alignments of inner and outer rings and pseudorings to deduce the intrinsic shapes and alignments of these features with respect to bars, to establish connections with specific resonances. For the remainder of this review I will summarize some of the morphological and statistical results from this catalog. In the following discussions, if an object is illustrated in the CSRG, the name is followed by CS. If illustrated in Buta \& Crocker (1991), the name is followed by BC.

\subsection{Outer Ring and Pseudoring Morphologies}

"OLR subclasses" - Perrine (1922) first pointed out the existence of a large diffuse outer ring around NGC 1291. Outer rings and pseudorings are symbolized by $(R)$ and $\left(R^{\prime}\right)$, respectively, preceding the type symbol in the de Vaucouleurs revised Hubble system. The morphology of outer rings and pseudorings in barred galaxies includes other subcategories that strongly resemble the gaseous rings and pseudorings that developed near the outer Lindblad resonance (OLR) in the test-particle simulations of Schwarz (1981; see Buta \& Crocker 1991). These are illustrated in Figure 2. Many barred galaxies display a pseudoring of type $\mathrm{R}_{1}^{\prime}$, defined by a $180^{\circ}$ winding of the spiral arms with respect to the ends of a bar or oval. Often, the pseudoring displays a blunt oval shape with "dimples" at the points where the arms return to the ends of the bar. ESO 287-56, shown in Figure 2, is an unusually strong example of this dimpling. At upper right in Figure 2, an "oval of Cassini" has been fitted to the outer pseudoring 
of ESO 287-56. This mathematical function (see Selby 1973) resembles the perpendicular-aligned OLR family of periodic orbits (see Schwarz 1981), and fits the ring well.

A substantial number of barred galaxies display a second type of feature known as an $\mathrm{R}_{2}^{\prime}$ pseudoring (see lower panels of Figure 2). In this type, the pseudoring is defined by an $\approx 270^{\circ}$ winding of the outer arms with respect to the bar ends, so that in two opposing quadrants the arm pattern is doubled. The pseudoring closes roughly along the line perpendicular to the bar in the galaxy plane, and dimpling is not generally seen. Detached true outer rings do not readily fit into these subcategories because the classification depends on the character of spiral arms. However, some true rings have a dimpled shape similar to $\mathrm{R}_{1}^{\prime}$ rings and are referred to as $\mathrm{R}_{1}$-type rings. The outer ring of NGC 1326 is an example (BC).

The $\mathrm{R}_{1}^{\prime}$ and $\mathrm{R}_{2}^{\prime}$ morphologies were predicted by Schwarz (1981) as the kinds of patterns that would be expected near the OLR in a barred galaxy. Each pattern is linked mainly to one of two major families of periodic orbits expected near the OLR. Their identification in large numbers of real galaxies is surely a noteworthy triumph for barred galaxy theory in its simplest, non-self-gravitating form. To add to the triumph is the identification of a rare combined pattern (not predicted by Schwarz) referred to in the CSRG as the $R_{1} R_{2}^{\prime}$ morphology, where the outer arms break not from the ends of the bar, but from an $\mathrm{R}_{1}$-type ring. An excellent large example is found in NGC 1079 (CS). The existence of this combined type, which may be linked to the population of both main families of OLR periodic orbits, provides some of the clearest evidence of the OLR in barred galaxy morphology.

The connection between these observed morphologies and the OLR is further strengthened by CSRG shape and alignment statistics. The $R_{1}$ and $R_{1}^{\prime}$ features are aligned perpendicular to the bar and have intrinsic axial ratios of $0.74 \pm 0.08$ on average, based on 187 CSRG galaxies. The $R_{2}^{\prime}$ features are aligned parallel to the bar and have intrinsic axial ratios of $0.87 \pm 0.06$, based on 135 CSRG galaxies. The alignments are consistent with the test-particle simulations. When a sample of barred galaxies is drawn from the CSRG, irrespective of outer feature subclass, the distribution of angles shows evidence of the presence of both alignments in the proportion 0.64:0.36. In general, outer rings and pseudorings in barred galaxies have an average intrinsic axis ratio of $0.82 \pm 0.07$, and most are aligned perpendicular to the bar.

Old-Population $R_{1}$ rings - Multicolor imaging of a large sample of CSRG outerringed galaxies led to the recognition of a subtle dichotomy in the morphology of such galaxies: the existence of $R_{1}$ rings which, though weak in blue light, are the dominant outer feature in the near-infrared. The prototype example is IC 1438 (CS), a large $R_{1} R_{2}^{\prime}$ case. The $R_{2}^{\prime}$ part is prominent in blue light, while the $R_{1}$ part is prominent in the near-infrared. The dichotomy suggests that the $R_{1}$ component formed first and left behind a stellar remnant (Byrd et al. 1994).

Other types of outer structures - Not all outer features in barred galaxies can be morphologically assigned to the OLR subclasses. Well-known s-variety barred spirals such as NGC 1300 and 1365 have outer spiral patterns which nearly close into other types of structures. That in NGC 1300 is intermediate in morphol- 


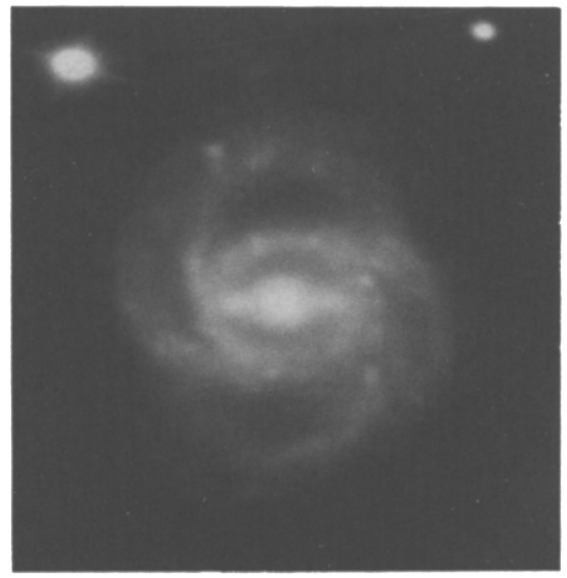

Figure 3. Deprojected $B$-band image of ESO 566-24, a symmetric four-armed ringed, barred spiral. Several field stars have been removed.

ogy between an $R_{1}^{\prime}$ pseudoring and an $R_{2}^{\prime}$ pseudoring, making this "prototype" barred spiral rather atypical.

Other barred galaxies have global multi-armed outer spiral patterns which can be connected to the OLR subclasses. An interesting example is found in ESO 566-24 (Figure 3 and BC). This object has four major spiral arms which break from an inner ring around the bar at points spaced by roughly $90^{\circ}$. The four arms intersect to form an outer pseudoring, and two of the arms wind similar to an $R_{1}^{\prime}$ pseudoring. Another interesting connection is found in the large, nearly face-on barred spiral NGC 3313 (CS). In blue light, the outer spiral arms of NGC 3313 are complex and extensive, showing a multiple character. In nearinfrared light, however, the outer multiple arms are very weak, and the pattern takes on the shape of an $R_{1}^{\prime}$ pseudoring. Since the multiple outer arms are at a much larger radius than this infrared feature, this galaxy provides a promising example of spiral structure extending well beyond the OLR.

Not all multi-armed patterns can be connected in these manners to an OLR subclass. Examples such as NGC 2307 (CS), 3450 (CS), and 3660 (CS) have outer arm patterns which do not resemble an OLR pattern at all. In the case of IC 5240 (CS), the spiral structure beyond a prominent inner ring is purely flocculent in the sense of the arm classes of Elmegreen \& Elmegreen (1987). Thus, it is important for any theory to explain the variety of spiral patterns associated with bars.

\subsection{Inner Rings and Pseudorings}

Inner rings in barred galaxies have a great range of morphologies. Most inner rings are pseudorings formed from spiral structure. Many examples are illustrated in Sandage \& Bedke (1994). Here I describe some interesting aspects of these features that help connect them to a specific resonance with the bar.

The CSRG galaxies NGC 6782 (CS) and ESO 325-28 (BC and Figure 2) display inner rings with distinct pointy oval shapes. The inner ring of NGC 3081 


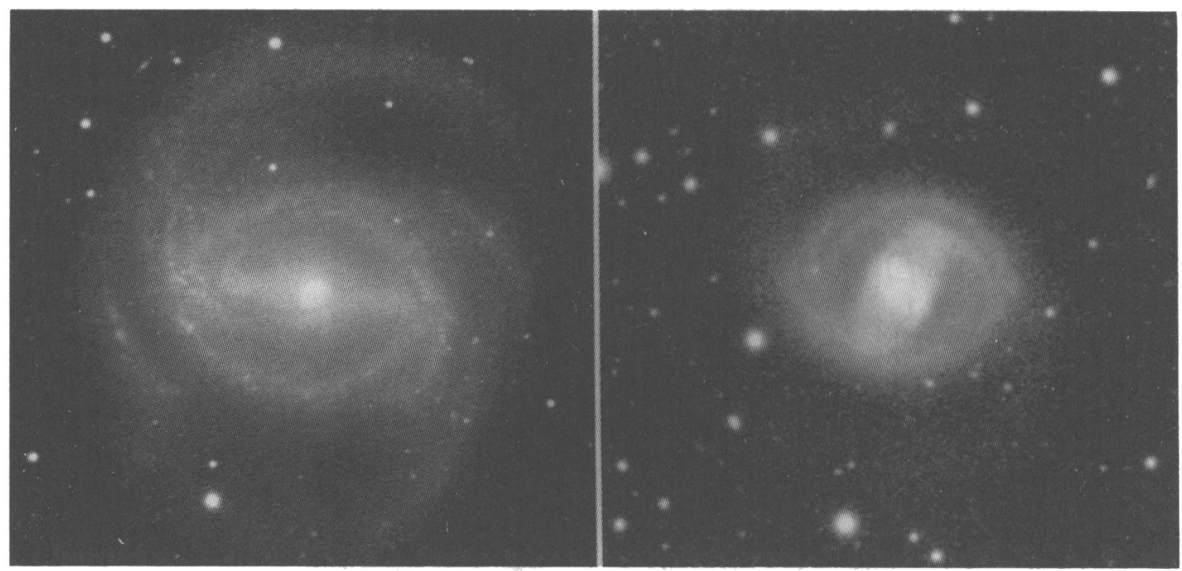

Figure 4. NGC 1433 (left), an aligned bar/inner ring galaxy, and ESO 565-11 (right), a misaligned bar/inner ring galaxy (both $B$-band)

(Buta 1990) is similar but is remarkable for its contrast. All of these rings have significant oval intrinsic shapes and enhanced blue colors near the major axis. Many other inner rings are made of wrapped spiral arms that encircle the bar closely. NGC 1433 (Figure 4, left panel) is an excellent example. If the arms were to close less tightly, we would have a pure s-variety barred spiral like NGC 1300 . The continuity of the variety characteristic is a fundamental aspect of the de Vaucouleurs revised Hubble classification system.

Statistics of apparent axis ratios and alignments of a large sample of inner SB rings from the CSRG reveal that these features have an average intrinsic axis ratio of $0.81 \pm 0.06$ and are aligned parallel to the bar. The alignment strongly links these features to the inner 4:1 resonance located just inside corotation. Test-particle models of barred spirals easily reproduce pointy oval shapes for a gaseous ring formed near this resonance (Simkin et al. 1980; Schwarz 1979; Byrd et al. 1994).

The most important finding about inner rings from the CSRG that is inconsistent with the test-particle models is intrinsic bar/inner ring misalignment. CSRG statistics imply that inner rings are aligned parallel to bars, but in spite of this there are nearly face-on objects in the catalog where the inner ring and the bar are misaligned by a substantial angle. For example, in ESO 565-11 (Figure 4 , right panel), the misalignment angle is $\approx 60^{\circ}$. Figure 4 compares ESO $565-11$ to an image of NGC 1433, a typical aligned bar/inner ring galaxy. The cause of misalignment is an intriguing problem, and verifying it further in other galaxies is an important issue for future work. The inner ring of ESO $565-11$ is near the rim of a prominent oval lens, making ESO 565-11 a misaligned bar/oval galaxy as well.

\subsection{Nuclear Rings, Pseudorings, and Bars}

Spectacular small rings of active star formation are seen in the centers of many spirals with bars or ovals. The rings average about $1 / 20$ th of the size of an 


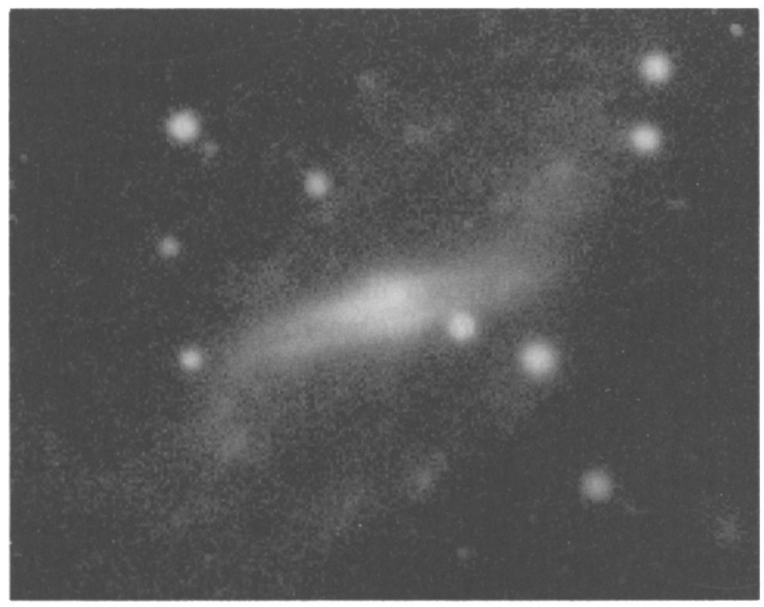

Figure 5. $\quad B$-band image of ESO 235-58, a false barred spiral?

outer feature, if present. The physically largest known example is found in the peculiar barred galaxy ESO 565-11 (Buta \& Crocker 1993a). The ring is $5 \mathrm{kpc}$ in diameter compared to the average of $1.5 \mathrm{kpc}\left(\mathrm{H}_{o}=75\right)$.

A wide range of morphologies is found for these features. The nuclear ring in ESO $565-11$ is defined mainly by 10 giant HII regions (Buta, Purcell, \& Crocker 1995) and has an intrinsic axis ratio of about 0.6. Prominent nuclear rings in other nearly face-on galaxies like NGC 1097, 1343, 3313, and 6782 are nearly round. Many nuclear rings have a spiral character, and most may prove to be spirals if imaged at high enough resolution. Some nuclear pseudorings or spirals could represent partly obscured nuclear rings or blue nuclei, as the dust lanes prominent in some bars can cross or arc around the rings. Nuclear rings also show a wide range of amplitudes and colors. Owing to the steep background luminosity gradient, a nuclear ring may only be prominent as a blue feature in a color index map. Not all nuclear rings are actively forming stars. Red nuclear rings of dust are also known.

Some galaxies have a small bar inside of a nuclear ring. An accurate judgment of the frequency of such secondary bars has not yet been made, but the phenomenon is likely to be common among early-type barred galaxies. The secondary bars are usually misaligned with the primary bars. It appears that any angle is possible (Buta \& Crocker 1993a; see also Friedli, these proceedings).

\subsection{False Barred Galaxies}

Finally, the CSRG turned up one very interesting example of a cataloged barred spiral which probably is not a barred spiral at all! The peculiar system ESO 235-58 (Figure 5) resembles a common late-type barred spiral, but the bar has a straight dust lane which divides the nucleus in a manner very atypical of bars. The dust lane is clearly visible on SRC field 235 and the photometric properties of the system were analyzed by Buta \& Crocker (1993b). The presence of the dust lane makes it likely that the apparent bar of ESO $235-58$ is really an edge- 
on disk galaxy, and that the system is related to polar ring galaxies. There are other similar cases of polar-ring-related systems being misclassified as barred spirals (e.g. NGC 660), but without kinematic data it is usually difficult to make an unambiguous identification.

\section{Conclusions}

Barred galaxy morphology has grown more interesting the past 15 years, and will likely become even more interesting as HST is used to observe the centers of such galaxies (see, for example, the paper by A. Barth, this conference). I believe that near-infrared observations are now essential to further probe barred galaxy morphology for new insights, and will provide some of the next important keys to understanding barred galaxy dynamics.

\section{References}

Athanassoula, E. 1992, MNRAS, 259, 345

Athanassoula, E. et al. 1990, MNRAS, 245, 130

Benedict, G. et al. 1992, AJ, 103, 757

Buta, R. 1984, PhD thesis, Univ. of Texas at Austin

Buta, R. 1990, ApJ, 351, 62

Buta, R. 1995, ApJS, 96, 39

Buta, R. \& Crocker, D. 1991, AJ, 102, 1715

Buta, R. \& Crocker, D. 1993a, AJ, 105, 1344

Buta, R. \& Crocker, D. 1993b, AJ, 106, 939

Buta, R., Purcell, G., \& Crocker, D. 1995, AJ, 110, 1588

Byrd, G. et al. 1994, AJ, 108, 476

Curtis, H. 1918, Pub. Lick Obs. 13, Part I, 12

de Vaucouleurs, G. 1956, Mem. Comm. Obs. No. 13

de Vaucouleurs, G. 1959, Handbuch der Physik, 53, 275

de Vaucouleurs, G. 1963, ApJS, 8, 31

Elmegreen, D. \& Elmegreen, B. 1987, MNRAS, 201, 1021

Hubble, E. 1926, ApJ, 64, 321

Hubble, E. 1936, The Realm of the Nebulae, New Haven: Yale Univ. Press

Kormendy, J. 1979, ApJ, 227, 714

Kormendy, J. 1981, in Structure and Evolution of Normal Galaxies, S. Fall \&

D. Lynden-Bell, Cambridge: Cambridge Univ. Press, 85

Kormendy, J. 1982a, in Morphology and Dynamics of Galaxies, L. Martinet \&

M. Mayor, Sauverny: Geneva Observatory, 113

Kormendy, J. 1982b, ApJ, 257, 75

Perrine, C. D. 1922, MNRAS, 82, 486

Sandage, A. 1961, The Hubble Atlas of Galaxies (Carnegie Inst. of Wash. Pub. No. 618 ) 
Sandage, A. \& Bedke, J. 1994, The Carnegie Atlas of Galaxies, Carnegie Inst. of Wash. Publ. No. 638

Selby, S. M. 1973, Standard Math. Tables, 21st ed., 394

Schwarz, M. 1979, PhD thesis, Australian National University

Schwarz, M. 1981, ApJ, 247, 77

Simkin, S., Su, H. J, \& Schwarz, M. P. 1980, ApJ, 237, 404

Vorontsov-Velyaminov, B. A. 1962-1968, Morphological Catalog of Galaxies (Moscow), Vols. 1-4.

\section{Discussion}

T. Hawarden: First, an observation: I haven't looked at as many galaxies on the sky surveys as you have, but I agree that I have never seen bar dust lanes with no offset. My question: have you ever seen a hexagonal ring which did not have pointed ends in projection, i.e., with flat ends as projected?

R. Buta: The best hexagonal features I know of are in NGC 7020 (inner ring/lens), NGC 4303 (inner pseudoring), and NGC 6753 (outer pseudoring). The features in the latter two are weakly hexagonal in the manner you described.

W. van Driel: The last galaxy you showed (ESO 235-58) reminds me of unpublished Westerbork HI line data of the NGC 3187/3190 pair. NGC 3187 looks like a barred spiral, but is actually edge-on with a tremendous warp perpendicular to the inner disk. I do not know of a straight dust lane through its nucleus.

R. Buta: ESO 235-58 is almost unique because it does have the splitting dust lane. This is not typical of polar ring galaxies or bars.

B. Elmegreen: Is there ever any evidence for ring structures at the outer $4: 1$ or outer $1: 1$ resonances?

R. Buta: Regarding simulations, I asked Phil Schwarz about the outer 4:1 resonance many years ago. His reply (Proc. ASA, 5, 464, 1984) was that the effect of the OLR is sufficient to prevent a ring from forming at the outer 4:1 resonance. I cannot say anything about the outer 1:1 resonance, but I believe we have identified an inner 1:1 resonance ring in the leading arm galaxy NGC 4622 (see AJ, 103, 1526, 1992). The galaxy has no bar, however. Not all CSRG galaxies fit into the conventional resonance ring categories, so I cannot say that other resonances are not represented.

B. Elmegreen: IC 2163 has an inner ring which is probably the transient result of an interaction with its massive neighbor NGC 2207. There is also an unaligned bar in IC 2163 but in this case it may be an illusion made by an offset nucleus seen in projection. Could other unaligned bar/inner ring galaxies be interacting?

R. Buta: My best case of intrinsic bar/ring misalignment is ESO 565-11, which does have some asymmetry but is not obviously interacting with another object. Other promising cases are definitely interacting: NGC 4319, for example. Although it would probably be easy to explain misalignment with an interaction (see, e.g., the models of Noguchi, 1987, MNRAS, 228, 635), we would have to explain what happened to the companion of ESO 565-11. 
M. Weinberg: A comment on the $\mathrm{R}_{1}, \mathrm{R}_{2}$, and detached rings. Most of our insight follows from gas forcing by a disturbance with constant $\Omega_{p}$. A nonconstant $\Omega_{p}$ will (or could) cause different fractions of trapping into the two resonances and might naturally explain detached rings. I saw this in my work in stellar kinematics (1994) and it might be worth looking for these effects in the gas response.

R. Buta: The great ring-forming test-particle simulations that I like to refer to so much really do not consider the effects of a variable $\Omega_{p}$. I agree that this is worth looking into.

$F$. Combes: What is the percentage of misaligned inner ring-bar galaxies? Have these misaligned galaxies some companions around?

R. Buta: The percentage must be fairly small because statistics of bar/ring position angles do not favor a significant population of inner rings with intermediate alignments. I cannot give an accurate figure on the percentage, however. Of the approximately 30 suspected cases of misalignment in the CSRG, several are members of small groups (e.g., NGC 619).

A. Zasov: Rings and related features (such as nuclear bars) may be found even in nonbarred galaxies. Can you summarize differences (if any) of their properties such as structure, shape, or size?

R. Buta: The rings of SA galaxies are certainly less well-understood than those in $\mathrm{SB}$ galaxies. On average, $\mathrm{SA}$ rings and pseudorings are intrinsically rounder than SB rings, and show a greater dispersion in linear sizes. Like SB rings, SA rings are generally sites of enhanced star formation. In most SA galaxies, it is likely that similar resonances are at work as in SB galaxies, but the nuclear, inner, and outer ring identifications may be less clearcut, as discussed in the CSRG. There are triple-ringed SA galaxies. However, the leading arm galaxy NGC 4622 suggests that resonances other than the inner 4:1 resonance, inner Lindblad resonance, and OLR may be at work in some SA galaxies.

$K$. Freeman: Some of the systems you showed with dimpled outer rings seem to have the inner and outer rings quite close together on the bar axis. Is this a problem for the identification of inner rings with the inner 4:1 resonance and the outer ring with the OLR: how could these resonances be so close, or is it just a projection effect?

R. Buta: The inner and outer ring come close together in such cases because the intrinsic major axis of the inner ring coincides with the intrinsic minor axis of the outer ring. It is not necessarily a projection effect. I do not think this is a problem for the above resonance identifications because the rings may be very oval. In this circumstance, the inner 4:1 resonant orbits and the inner family of OLR orbits may come close together on the bar axis.

$P$. Teuben: Hexagonal rings: are they due to the $6: 1$ resonance?

R. Buta: This is certainly the most likely explanation of the hexagonal feature seen in NGC 7020. But I must say that pending further observations and modeling, this is only speculation. 